\title{
The New Emerging Science of Cardio-Oncology
}

\author{
Hisham Ben Lamin* and Abdulkafi Toshani \\ Cardio-Oncologist, Spain
}

Submission: November 17, 2017; Published: November 21, 2017

*Corresponding author: Hisham Ben Lamin, Clinical Cardiologist, Cardio-Oncologist, Priego de Cordoba Spain, Email: hishambenlamin@gmail.com

\section{Opinion}

Cardio-Oncology is a new science, wide, and involves Cardiologists, Oncologists, Pathologists, Hematologists, Pharmacologists and Nurses. There are some principles we should all know in dealing with this science to get the best results and benefits to our patients: Cardiologists should know some Oncology basics and Oncologists to know some Cardiology basics. Cancer itself without administration of cancer therapy can cause Cardio toxicity and lead to left ventricular dysfunction and heart failure and here we need also Cardiologists to stand beside the Oncologists. We have to know that we are dealing with two types of individuals, those with an established cardiovascular diseases and going to receive cancer medications which may worsen their CV status, and the group which is without obvious cardiovascular disease but will receive cancer drugs that may cause them develop a new CV diseases. The cardio toxicity will not only affect the Cardiomyocytes leading to $\mathrm{LV}$ dysfunction and heart failure but also affects the kidneys, brain, veins and arteries. A new state of hypertension may start or a worsening of an existing one. It is important to know that we are mainly using 3 types of therapies in treating cancer, the Chemotherapy, the Tar gating agents and radiotherapy. Other emerging therapies are now approved such as immunotherapy. The genetic element and susceptibility of individuals are an important issue, there will be patients receiving high accumulating doses of anthracyclines without showing serious complications and others receiving low doses and ending up with real complications and CV events.

The knowledge of cardio toxic cellular biology is a must to know to all cardiologists, this will help much in knowing how to use these agents. The Cardio-Oncology clinics should consist of 3 types of clinicians, the Cardiologists, the Oncologists and the Nephrologists and all patients should be assessed by the 3 physicians and data should be circulated by electronic recording among them. The patients should be assessed pretreatment, during treatment, at the end of treatment and followed up as needed and this may reach till 3 decades after the end of treatment. The control and assessment should start from childhood survivors ending up in elderly and old age individuals, and with each age, we have to know the characteristics of the disease complications and manage them properly and accordingly. Each time, the risk factors should be in mind: Gender (females more than males), age (less than 15 and more than 65 years), anthracyclines regimen and total dose given, mediastinal or spinal radiation including total dose given and amount of heart tissue radiated, putting in mind always that radiotherapy is not only accelerating atherosclerosis but also causing pericarditis, myocarditis, endocarditis and arrhythmias.

Diagnosis of early cardiac damage in individuals on cardiotoxic agents and radiation is important and life saving and tools used should include proper history taking, baseline and serial ECG monitoring, Echocardiography in the form of 2D, TDI and GLS (LV regional deformation). Other imaging like CMR, CT scanning can support. Biomarkers like troponin to detect cardiomyocytes injury and BNP to detect volume and pressure overload in LV (diastolic dysfunction) can help.

If cardio-toxicity takes place we have to prepare ourselves to manage the LV dysfunction, heart failure, Ischaemic heart disease, acute coronary syndrome, PHT, arrhythmias and prolonged QTc complications, thrombosis and thromboembolism, hypertension and its complications, and radiotherapy adverse effects. All physicians should use the schemes and logarithms for follow up of each agent used, anthracyclines, targated therapy and radiotherapy. Special attention should be given to the new emerging agents called the angiogenesis inhibitors causing several toxicities including severe hypertension that may not be responding to medical management. Cytotoxic agents may induce atrial fibrillation, like in Cisplatin or in 5FU treatment and we should be ready for managing that. Some chemotherapeutics cause angina and ischaemic heart disease and may end up in myocardial infarction (5FU).

Details of the HER2 targated therapy and tyrosin kinase inhibitors should be known in details due to their clinical and therapeutic importance, this knowledge should include the 
molecular biology knowledge. Immuno-therapies are now approved by the FDA and they are in the market now, very important, giving much hope, they include the following: therapeutic cancer vaccines, monoclonal antibodies, checkpoints inhibitors and cytokines. Novel agents are coming soon like P13k I, mTOR inhibitors, Ubiquity, FGF, RAS ERK inhibitors ...etc. Treatment of cardio-toxicity should be at a very early stage to save the heart from lethal complication that may occur; this may need the new imaging technique of Global Longitudinal Strain GLS which will detect the early changes in the left ventricle (regional deformation) at least 3 months earlier than the changes in ejection fraction EF. Treatment of the LV dysfunction should be based on ACE inhibitors and beta blockers, other evolving complications like hypertension, renal insufficiency, heart failure, angina, thrombosis /thromboembolism, myocardial infarction etc should be managed as per the guidelines.

New guidelines for managing cancer patients in the cath. Lab are in hand now and should be well known by interventional cardiologists. Cardiology consultation should be seeked if the LVEF is going below 53\% or GLS is below limit of normal or troponin levels are elevated. Follow up recommendations: patients treated with doxorubicin or other type 1 agents should be followed up at the completion of therapy for doses below $240 \mathrm{mg} / \mathrm{m} 2$ and before each additional cycle after exceeding $240 \mathrm{mg} / \mathrm{m} 2$. For patients treated with trastuzumab or other type II toxicity agents should be followed up by echocardiography every 3 months during therapy, if trastuzumab is following anthracyclines regimen, the baseline evaluation should include EF, GLS and troponin and repeated every 3 months during treatment and 6 months after treatment. If tyrosin kinase inhibitors are used like sunitinib or sorafenib in patients with $\mathrm{CAD}$, it is a must to monitor blood pressure and symptoms closely; the recommendation is to follow up at one month and every 3 months while on therapy. Sub-clinical LV dysfunction should be monitored during chemotherapy by an abnormal GLS or elevated troponin levels. Evaluation of patients in the pre treatment, during treatment and after treatment stages in details should be known by heart in all managing physicians.

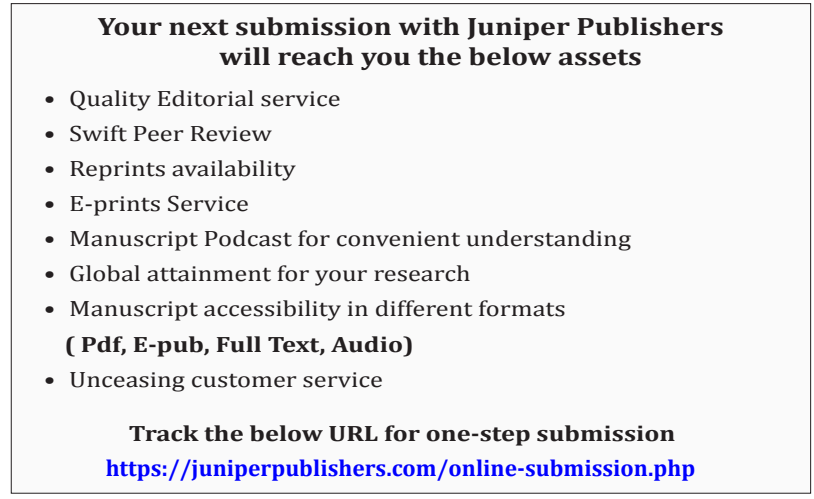

